\title{
Role of Epac1, an Exchange Factor for Rap GTPases, in Endothelial Microtubule Dynamics and Barrier Function
}

\author{
Seema Sehrawat, ${ }^{*+}$ Xavier Cullere, ${ }^{*+}$ Sunita Patel, ${ }^{\ddagger}$ Joseph Italiano, Jr., ${ }^{\ddagger}$ \\ and Tanya N. Mayadas*
}

*Center of Excellence in Vascular Biology, Department of Pathology, and \$Translational Medicine Division,
Department of Medicine, Brigham and Women's Hospital and Harvard Medical School, Boston, MA 02115

Submitted November 1, 2006; Revised December 10, 2007; Accepted December 26, 2007

Monitoring Editor: Asma Nusrat

\begin{abstract}
Rap1 GTPase activation by its cAMP responsive nucleotide exchange factor Epac present in endothelial cells increases endothelial cell barrier function with an associated increase in cortical actin. Here, Epac1 was shown to be responsible for these actin changes and to colocalize with microtubules in human umbilical vein endothelial cells. Importantly, Epac activation with a cAMP analogue, 8-pCPT-2' O-Me-cAMP resulted in a net increase in the length of microtubules. This did not require cell-cell interactions or Rap GTPase activation, and it was attributed to microtubule growth as assessed by time-lapse microscopy of human umbilical vein endothelial cell expressing fluorophore-linked microtubule plus-end marker end-binding protein 3. An intact microtubule network was required for Epac-mediated changes in cortical actin and barrier enhancement, but it was not required for Rap activation. Finally, Epac activation reversed microtubuledependent increases in vascular permeability induced by tumor necrosis factor- $\alpha$ and transforming growth factor- $\beta$. Thus, Epac can directly promote microtubule growth in endothelial cells. This, together with Rap activation leads to an increase in cortical actin, which has functional significance for vascular permeability.
\end{abstract}

\section{INTRODUCTION}

Cell-cell interactions are critical for maintaining a patent endothelial cell monolayer that regulates fluid passage and leukocyte egress into the extravascular space. Endothelial cell junctions are tightly controlled to allow these two important endothelial functions to proceed during an inflammatory response without disruption of the endothelial integrity. The small GTPases of the Rho family are involved in regulating both vascular leakage and leukocyte transmigration through effects on the cytoskeleton (van Hinsbergh and van Nieuw Amerongen, 2002; Wittchen et al., 2005a). Elevation of cAMP in endothelial cells has been long recognized to increase barrier function under basal conditions and to reverse vascular leakage by inflammatory mediators through activation of protein kinase A (PKA) (Yuan, 2003). Recent work by us and other groups suggests that activation of Rap GTPases, a member of the Ras family of GTPases, through their cAMP-responsive guanine exchange factors (GEFs) present in endothelial cells, Epac1 and -2 , significantly increased barrier function. This was associated with an increase in cortical actin and the subsequent increase in the continuity of VE-cadherin staining

This article was published online ahead of print in $M B C$ in Press (http:/ / www.molbiolcell.org/cgi/doi/10.1091/mbc.E06-10-0972) on January 2, 2008.

+ These authors contributed equally to this work.

Address correspondence to: Tanya N. Mayadas (tmayadas@rics. bwh.harvard.edu).

Abbreviations used: EB3, end-binding protein 3; F, forskolin; GEF, guanine exchange factor; HUVEC, human umbilical vein endothelial cell; MT, microtubule; O-Me-cAMP, 8-pCPT-2'O-Me-cAMP; R, rolipram; TER, transelectrical resistance. at endothelial cell borders (Cullere et al., 2005; Fukuhara et al., 2005; Kooistra et al., 2005; Wittchen et al., 2005b). Furthermore, Epac activation reversed thrombin (Cullere et al., 2005) and vascular endothelial growth factor (Fukuhara et al., 2005) induced permeability and in the former was associated with down-regulation of Rho GTPase (Cullere et al., 2005). The Epac-induced increase in barrier function occurred through a PKA-independent pathway, thus introducing a new, alternate cAMP-inducible pathway of barrier regulation. However, the mechanisms linking Epac/Rap to changes in cortical actin and subsequent barrier enhancement remain unclear.

In addition to the actin remodeling, microtubule (MT) dynamics control fundamental cellular functions, such as cell shape, polarity, motility, and division, as well as the delivery of factors to specific cell compartments. MTs are highly dynamic structures that grow and shrink, and they provide polarized tracks along which motor proteins move. The cycle of growth and shortening of plus ends during dynamic instability may provide a means for MT to explore peripheral structures such as focal adhesions and thus regulate their maturation (Small and Kaverina, 2003; Gundersen et al., 2004). There is increasing evidence that MTs also regulate the dynamics of cell-cell junctions. $\beta$-Catenin, a component of adherens junctions (AJs), has been shown to bind the MT-motor dynein (Ligon et al., 2001), whereas p120 catenin directly interacts with MTs (Yanagisawa et al., 2004), thus providing a direct link between AJs and the MT network. In endothelial cells, the MT network has been shown to play a role in vascular permeability. MT disruption with nocodazole promotes barrier dysfunction, which is attenuated by pretreatment with forskolin (Birukova et al., 2004c). Furthermore, tumor necrosis factor (TNF), thrombin, and transforming growth factor (TGF)-induced endothelial permeability is associated with a decrease in stable tubu- 
lin and destabilization of the peripheral MT network (Petrache et al., 2003; Birukova et al., 2004a, 2005). As with actin remodeling, members of the Rho GTPase family regulate MT dynamics, and they play specific roles in MT rearrangement (Watanabe et al., 2005). cAMP has been long known to promote the assembly of MTs and microfilaments (Seite et al., 1977). Recent in vitro data suggest that Epac1 and -2 interact with the light chain 2 of microtubule-associated protein MAP1A (Yarwood, 2005), which promotes nucleation and elongation of tubulin (Noiges et al., 2002). Here, we tested the hypothesis that Epac modulates MT dynamics, which couples to the actin cytoskeleton in endothelial cells to modulate barrier function.

\section{MATERIALS AND METHODS}

\section{Reagents}

The antibodies used were rabbit anti-Rap1, rabbit anti-Rap2, goat anti-Epac1, and goat anti-Epac2 (Santa Cruz Biotechnology, Santa Cruz, CA); anti-nonmuscle myosin, tubulin, and $\beta$-actin (Sigma-Aldrich, St. Louis, MO); phospho-myosin light chain (MLC) 2 (Ser19) and phospho-c-Jun $\mathrm{NH}_{2}$-terminal kinase (JNK) (Cell Signaling Technology, Beverly, MA); and anti-VE-cadherin (cadherin-5) (Beckman Coulter, Fullerton, CA). 8-pCPT-2' O-Me-cAMP (OMe-cAMP) was from Biolog Life Science Institute (Bremen, Germany). Human TNF- $\alpha$ (biological activity of $2 \times 10^{7} \mathrm{U} / \mathrm{mg}$ ) and TGF- $\beta 1$ were from R \& D Systems (Minneapolis, MN). 4,6-Diamidino-2-phenylindole (DAPI), phalloidin, nocodazole, Taxol, ATP, and cytochalasin D were from Sigma-Aldrich.

\section{Cell Culture}

Human umbilical vein endothelial cells (HUVECs) were isolated and cultured on gelatin-coated dishes (Cullere et al., 2005). Human pulmonary aortic endothelial cells (HPAEC) were purchased from Clonetics (San Diego, CA). Cells were treated as follows: $2 \mu \mathrm{M}$ cytochalasin D, $0.15 \mu \mathrm{M}$ nocodazole, 100 $\mu \mathrm{M}$ O-Me-cAMP, $10 \mu \mathrm{M}$ Taxol (paclitaxel), $10 \mu \mathrm{M}$ forskolin, $20 \mu \mathrm{M}$ rolipram, $2 \mathrm{ng} / \mathrm{ml}$ TGF- $\beta 1$, or $20 \mathrm{ng} / \mathrm{ml}$ TNF. Live-cell movies of end-binding protein 3 (EB3)-green fluorescent protein (GFP) were obtained from cells that were starved overnight in M199 medium containing $0.1 \%$ bovine serum albumin.

Plasmid DNA and small interfering RNA (siRNA) duplexes were introduced into HUVECs using Amaxa Nucleofector Technology (Amaxa, Koeln, Germany), and cells were examined after $48 \mathrm{~h}$. HUVEC were used 48 and $24 \mathrm{~h}$ after retroviral and adenoviral transduction, respectively, with infection efficiencies ranging from $\sim 75$ to $100 \%$, as determined by GFP fluorescence.

\section{Plasmids and Virus Production}

Tubulin cDNA was excised from GFP-tubulin (BD Biosciences, San Jose, CA), and it was cloned into the retroviral vector pLEGFPC1. Epac1-yellow fluorescent protein (YFP) cDNA (Mei et al., 2002) was provided by Dr. Xiaodong Cheng (University of Texas, Galveston, TX) and GFP cDNA (pEGFPN3) was from BD Biosciences. EB3-GFP cDNA was provided by Dr. Neils Galjart (Department of Cell Biology and Genetics, Erasmus MC, Rotterdam, The Netherlands), and it was cloned into the pWZL retroviral vector. Retrovirus expressing these constructs were generated, and they were used in HUVECs as described previously (Cullere et al., 2005; Patel et al., 2005). Rap1GAPexpressing adenovirus (Wittchen et al., 2005b; gift from Dr. Peter J. Casey, (Duke University, Durham, NC), containing the Rap1GAP-IRES-GFP bicistronic cassette, was propagated in human embryonic kidney (HEK) 293 cells, and high titer stocks were obtained using Adeno- $X$ virus purification kits (BD Biosciences)

\section{Cell Fractionation}

Triton X-100 fractionation was conducted essentially as described previously (Borbiev et al., 2001). Briefly, confluent endothelial cells (80-100\%) were fractionated into Triton-soluble and -insoluble cytoskeletal fractions. Cells were incubated in ice-cold extraction buffer (25 mM HEPES, pH 7.4, $150 \mathrm{mM}$ $\mathrm{NaCl}, 3 \mathrm{mM} \mathrm{MgCl}, 5 \mathrm{mM}$ EDTA, 0.5\% Triton X-100, $25 \mathrm{mM}$ NAF, $1 \mathrm{mM}$ $\mathrm{Na}_{3} \mathrm{VO}_{4}, 10 \mathrm{mM}$ sodium pyrophosphate, and protease inhibitor cocktail [Calbiochem, San Diego, CA]) with agitation for $5 \mathrm{~min}$ at $4^{\circ} \mathrm{C}$. The Triton-soluble fraction was collected after centrifugation. The remaining pellet (cytoskeletal fraction) was treated with SDS extraction buffer (0.5\% Triton X-100, 0.5\% SDS, $10 \mathrm{mM}$ Tris- $\mathrm{HCl}, \mathrm{pH} \mathrm{6.8,} \mathrm{and} \mathrm{protease} \mathrm{inhibitor} \mathrm{cocktail).} \mathrm{Samples} \mathrm{were}$ subjected to SDS-polyacrylamide gel electrophoresis (PAGE) and Western Blot analysis.

\section{SiRNA}

HUVECs were electroporated with $600 \mathrm{nM}$ luciferase (Luc) or Epac1-specific siRNA oligonucleotides (QIAGEN, Valencia, CA) using protocols recommended by Amaxa Biosystems (Gaithersburg, MD). The sequences of the
Epac1 and luciferase siRNA used were as follows: Epac 1-1, 5'-AACTCGGTGAAGCGAGAATTA-3'; Epac 1-2, 5'-AAGGGCACTTCGTGGTACATT-3'; Epac 1-3, 5'-AAGTACCACCTTAGCTCTAT-3'; and luciferase, 5'-CGUACGCGGAAUACUUCGATT-3'

\section{Rap Activation Assays}

Rap pull-down assays were performed as described previously (Cullere et al. 2005), and samples were subjected to SDS-PAGE and Western blot analysis by using anti-Rap1 and Rap2.

\section{Western Blot Analysis and Immunofluorescence}

Endothelial cell proteins were separated using 4-20\% gradient SDS-PAGE gels, transferred to nitrocellulose, and immunoblotted with primary antibodies using standard protocols. For immunofluorescence, HUVECs were plated on fibronectin-coated glass coverslips (Fisher Scientific, Pittsburgh, PA), and processed as described previously (Cullere et al., 2005).

\section{Quantification of Microtubule Length}

Changes in MT length were analyzed using the process extension application from MetaMorph software, version 6.1 (Molecular Devices, Sunnyvale, CA) used previously for analysis of neurite extension (Tudor et al., 2005). Cells were stained with anti- $\beta$-tubulin, and DAPI to visualize nuclei. Images were captured with a Leica microscope using the $20 \times$ lens. Collected data were exported and processed using Adobe Photoshop software (Adobe Systems, Mountain View, CA). Process extension analysis was performed on eight to 10 randomly chosen fields per group. MT length was defined as the traced distance from the MT tip to the point where MT emanated from the nucleus (determined by DAPI staining). To obtain an average length (mean process length) per cell, the mean outgrowth for all the cells per field was divided by the mean number of processes.

\section{Live-Cell Imaging of EB3-GFP}

HUVECs plated on glass coverslips and infected with EB3-GFP retroviral supernatants were mounted on a Nikon Eclipse TE-2000E microscope, and they were imaged essentially as described previously (Patel et al., 2005). Live-cell movies of EB3 rockets were recorded, and rates of rocket movement were measured for 15-20 rockets per cell.

\section{Measurement of Endothelial Transelectrical Resistance}

Electrical resistance across the EC monolayer was measured using an electrical cell impedance sensor system (Applied Biophysics, Troy, NY). Cells were grown on gold microelectrodes $\left(10^{-3} \mathrm{~cm}^{2}\right)$ in polycarbonate wells. All electrical resistance data are presented as normalized values. Briefly, current was applied across the electrodes by a $4000-\mathrm{Hz}$ AC voltage source with an amplitude of 1 . Resistance values from each microelectrode (measured in ohms) were normalized as the ratio of measured resistance to baseline resistance, and they were plotted versus time.

\section{Statistical Analysis}

Results are expressed as means \pm SD. Stimulated samples were compared with controls by unpaired Student's $t$ test. The data on EB3-GFP rates and fold induction were analyzed using a standard one-way analysis of variance (ANOVA) with the Holm-Sidak method. $\mathrm{p}<0.05$ was considered statistically significant.

\section{RESULTS}

\section{Activation of Epac1 Increases Cortical Actin and Is Required for Barrier Integrity}

Previous studies showed that treatment of HUVEC monolayers with the cAMP analogue O-Me-cAMP that specifically activates Epac proteins resulted in an increase in cortical actin as assessed by immunofluorescence staining. O-Me-cAMP activates both Epac1 and Epac2. HUVECs express Epac1. HUVECs also showed immunoreactivity to an Epac2 antibody (Cullere et al., 2005). Epac2 levels may vary depending on the culturing conditions or treatment of HUVECs as it is detected in HUVEC microarrays (RapGEF4; Chi et al., 2003), and it was shown to be inducible in human microvascular endothelial cells (Hong et al., 2007). However, because Epac2 RNA and protein were not detected in HUVECs by two groups (Kooistra et al., 2005; Fang and Olah, 2007), we examined Epac2 message by reverse transcription-polymerase chain reaction analysis. We observed Epac1 but a lack of Epac2 in unstimulated HUVECs (Sup- 
plemental Figure 1S). This suggests cross-reactivity of the Epac2 antibody with a different protein in our previous study (Cullere et al., 2005), and led us to focus on evaluating the role of Epac1, the primary Epac isoform in HUVECs, in $\mathrm{O}-\mathrm{Me}-\mathrm{cAMP}$-induced changes in cortical actin and barrier function. Epac1 siRNA in HUVECs resulted in a $>90 \%$ reduction in Epac1 protein compared with cells expressing a control (Luc) siRNA (Figure 1A). Epac1 siRNA-expressing HUVECs failed to increase cortical actin in response to treatment with O-Me-cAMP compared with cells expressing control Luc siRNA (Figure 1B). Similarly, the increase in cortical actin upon cAMP elevation with forskolin $(\mathrm{F})$ and rolipram

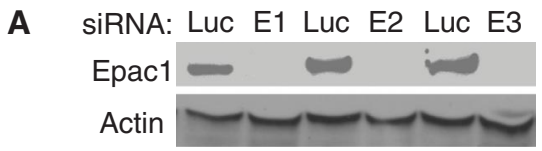

B

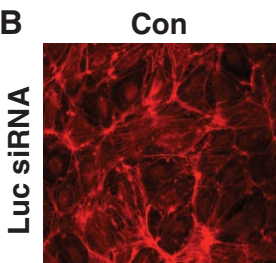

+O-Me-cAMP
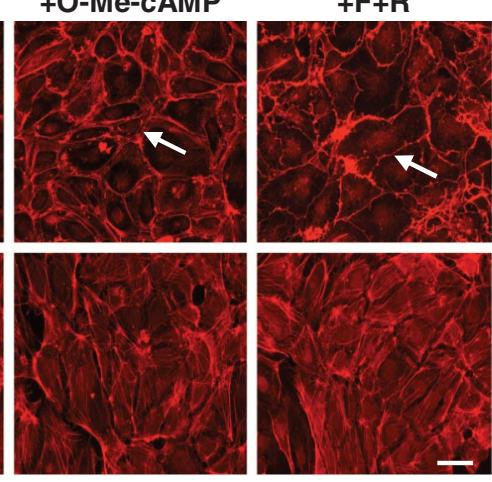

C
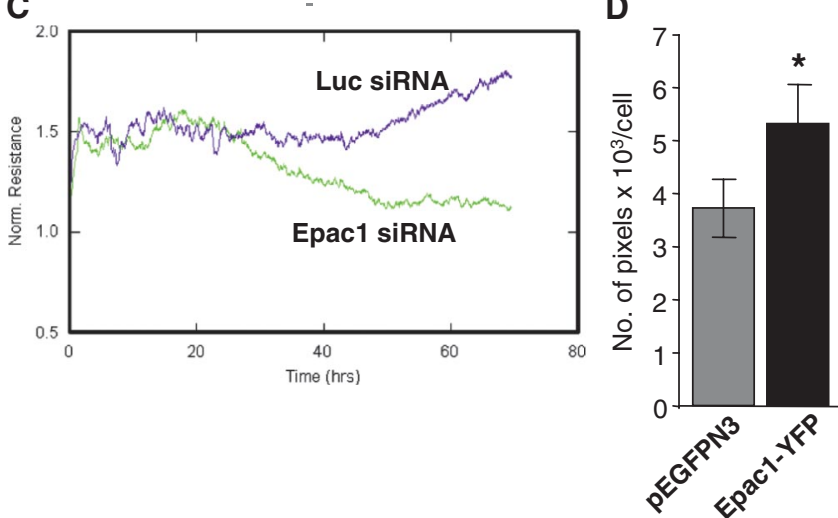

Figure 1. Analysis of F-actin after Epac activation and Epac1 knockdown in HUVECs. (A) HUVECs transfected with control (Luc) or one of three Epac1 siRNA (E1-E3) were analyzed for Epac1 and $\beta$-actin protein levels by Western blot. (B) Luc and E1 siRNAexpressing cells were treated with O-Me-cAMP or F + R for $20 \mathrm{~min}$, and then they were stained with rhodamine-phalloidin to visualize F-actin. O-Me-cAMP and $\mathrm{F}+\mathrm{R}$ increased cortical actin (white arrows) in Luc siRNA- but not Epac1 siRNA-expressing cells. Similar changes in actin localization were obtained with E2 and E3 siRNA (data not shown). The degree of phenotype seen relates to the amount of Epac knockdown. The set of images shown are representative of dramatic silencing of Epac. Bar, $10 \mu \mathrm{M}$. (C) HUVECs were transfected with Luc and Epac1 siRNA, and changes in transendothelial resistance (permeability) were analyzed over time. (D) Cells were transfected with vector expressing GFP (pEGFPN3) or Epac1-YFP, and cell size was determined by evaluating the number of pixels per cell. Twenty-five cells per group were evaluated. One of two to three representative experiments is shown for each panel. * $\mathrm{p}<0.05$.
(R) was observed in Luc siRNA but not Epac1 siRNA-expressing cells (Figure 1B). Transelectrical resistance (TER), measured using an electrical cell-substrate impedance sensing system, allowed a time-course analysis of the endothelial response to Epac1 siRNA expression. Silencing of Epac1 resulted in an increase in vascular permeability (Figure 1C). It is noteworthy that the -fold reduction in TER in Epac1silenced cells is not as pronounced as with edemagenic agents, such as TNF- $\alpha$ and TGF $\beta$ (Figure 6, E and F). Overexpression of YFP-tagged Epac1 (Epac1-YFP) in HUVECs resulted in an increase in cell spreading compared with HUVECs expressing GFP-vector alone (Figure 1D). The enhanced cell spreading is likely the result of Epac1 responding to endogenous levels of cAMP in the endothelial cell monolayer to promote integrin-dependent adhesion and spreading. This explanation is supported by data reporting that Epac overexpression is capable of activating Rap1 in the absence of cAMP-elevating agents (de Rooij et al., 1998; Kawasaki et al., 1998). Together, these data suggest that Epac1 plays a major role in the observed cAMP-dependent cytoskeletal changes in HUVECs, and this has consequences for endothelial barrier function.

\section{Epac1 Colocalizes with Microtubules and Its Activation Leads to Changes in the Microtubule Network}

High-power microscopic examination of Epac1-YFP-transfected HUVECs revealed Epac1 in filamentous MT-like structures (Figure 2A), a distribution present both before and after O-Me-cAMP treatment. Confocal microscopic analysis of HUVEC monolayers transduced with Epac1-YFP or GFP vector and immunostained for $\beta$-tubulin revealed colocalization of Epac1 with $\beta$-tubulin-containing structures (Figure 2B). In addition to the localization of Epac with the distal microtubules, colocalization with the microtubuleorganizing center (MTOC) (arrowhead) was also observed, which strengthens our conclusion that Epac1 associates with the MT network. Next, we evaluated whether activation of Epac1 in HUVEC monolayers resulted in changes in MT organization. HUVEC treatment with O-Me-cAMP led to the elongation of the MTs toward the cell periphery and to increased delineation of individual filaments (Figure 2C). To further confirm this observation, human pulmonary artery endothelial cells were transduced with GFP- $\beta$-tubulin, and the cells were imaged before and after stimulation with $\mathrm{O}-\mathrm{Me}-\mathrm{cAMP}$. Consistent with the results in HUVECs, O-MecAMP treatment resulted in the elongation of GFP-tubulinexpressing MTs in human pulmonary aortic endothelial cells (Figure 2C).

\section{Epac Plays a Role in Endothelial Microtubule Dynamics}

To evaluate whether Epac activation resulted in a productive increase in MT lengths, MT process lengths were quantitated using MetaMorph software developed previously to measure neurite process extension (Woo and Gomez, 2006). $\mathrm{O}-\mathrm{Me}-\mathrm{cAMP}$ treatment of confluent monolayers resulted in a significant increase in mean process length (Figure 3A). Treatment with the MT-disrupting agent nocodazole significantly reduced process lengths in unstimulated cells, confirming the MT dependence of this analysis (data not shown). Junctional complexes may serve as activation points for capturing and stabilizing growing MTs. Thus, the dependence of MT extension by O-Me-cAMP on junctional complexes was evaluated by assessing the effect of the compound on confluent monolayers under calcium-depleting conditions (Figure 3A) that result in the loss of VE-cadherin at cell-cell contacts (Cullere et al., 2005). O-Me-cAMP treatment led to an increase in MT length that was similar in 
A
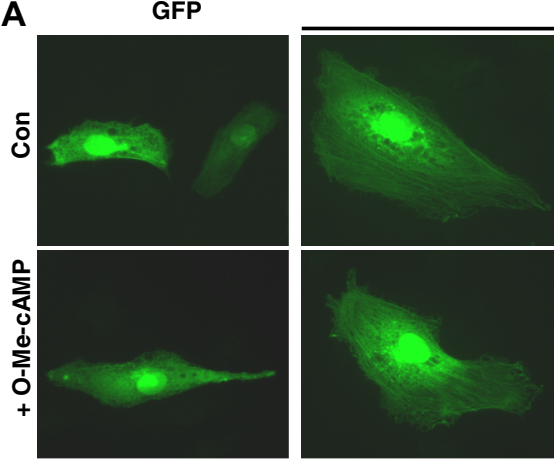

YFP-Epac1

B
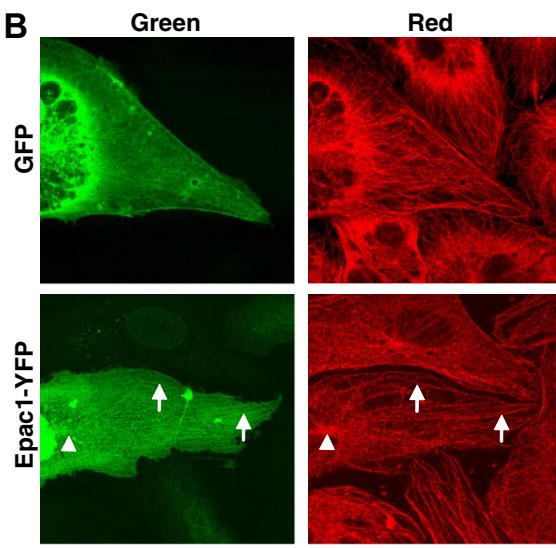

C

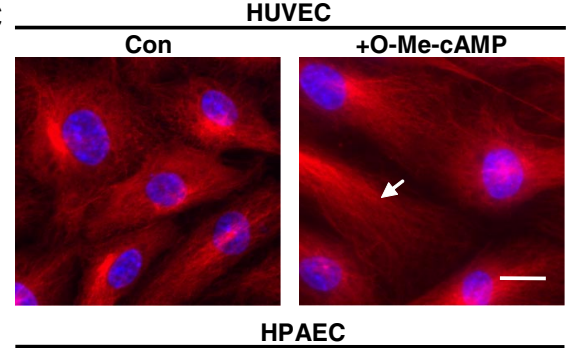

Con

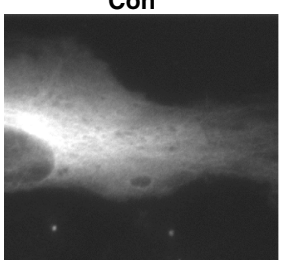

+O-Me-cAMP

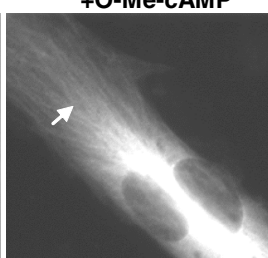

Figure 2. Epac-1 colocalizes with $\beta$-tubulin in HUVECs and Epac activation affects microtubule distribution. (A) HUVECs retrovirally transduced with GFP alone ( $a$ and $b$ ) or YFP-Epac1 $(\mathrm{c}-\mathrm{f})$ were untreated (Con) or incubated with O-Me-cAMP. e and $\mathrm{f}$ are magnified images of $\mathrm{c}$ and $\mathrm{d}$. YFP-Epac1 was present in a filamentous distribution (arrows). YFP-Epac1 expression resulted in cleavage of the heterologous protein as assessed by Western blot analysis (data not shown), which may account for the observed nuclear and background fluorescence (typically observed with YFP or GFP alone) in YFP-Epac1-expressing cells. Bar, 10 $\mu \mathrm{M}$. (B) $\beta$-Tubulin staining of GFP- and YFP-Epac1-transduced HUVECs stimulated with O-Me-cAMP and subjected to confocal microscopy. Colocalization of YFP-Epac1 signal (green) with $\beta$-tubulin (red) in filaments (arrows) and the MTOC (arrowhead) from which MTs radiate was observed (Bar, 3-5 $\mu \mathrm{m}$ ). (C) Immunofluorescence of HUVECs stained with anti $\beta$-tubulin antibody or HPAECs expressing GFP-tubulin treated without (Con) or with O-Me-cAMP. HUVECs contain a fine array of microtubules that elongate and extend to the periphery of the cell after
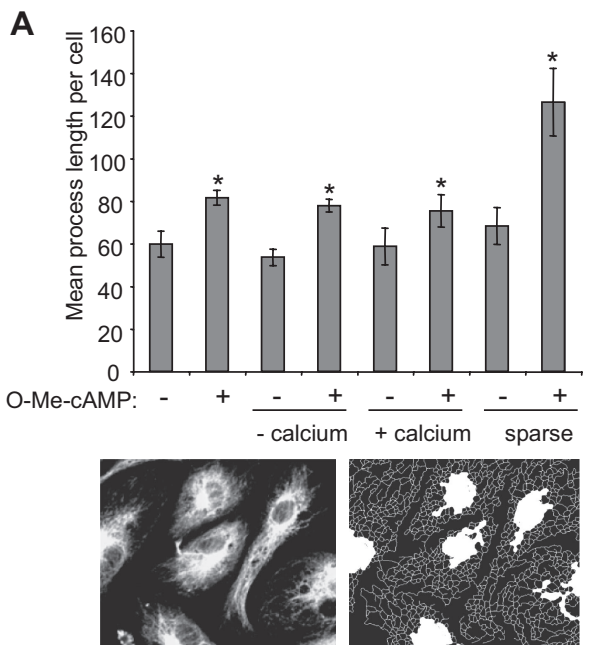

B

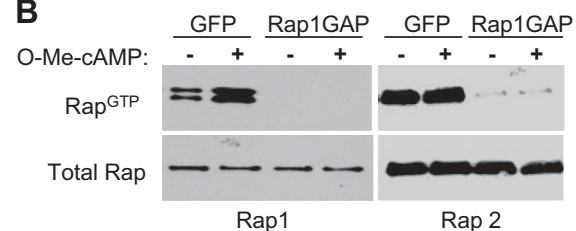

C

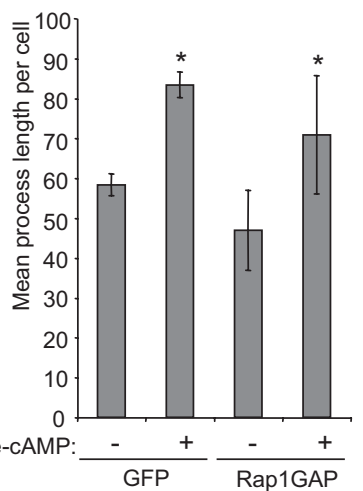

Figure 3. O-Me-cAMP treatment results in an increase in MT length that is independent of cell-cell junctions and Rap activation. (A) HUVECs were treated with $(+)$ or without $(-)$ O-Me cAMP cultured in complete medium, low calcium medium (- calcium), and after calcium replenishment (+ calcium), and complete medium but cultured under sparse conditions (sparse). MT distribution was quantitated using morphometric analysis of monolayers stained with anti- $\beta$-tubulin antibody. Data are expressed as the mean process length per cell. Bottom, on the left is a black and white image of an anti-tubulin and DAPI-stained sample that was examined. On the right is an image of MT structures, visualized as white tracks, detected by the MetaMorph software. The DAPI signal and immediately surrounding signal are saturated so as to demonstrate the fine MT structures detected by the software. (B) HUVECs were transduced with GFP control and Rap1GAP-GFP adenoviruses. Pull-down assays to detect active Rap1 and 2 (Rap GTP ) were performed. Western blot of total Rap1 and Rap2 (total Rap) shows equivalent levels of Rap in all samples. Shown is one representative of three independent experiments. (C) Mean process length was quantified in transduced cells treated with $(+)$ or without $(-)$ O-Me-cAMP. Data shown in A and C are from three independent experiments. ${ }^{*} \mathrm{p}<0.05$ compared with the associated $(-)$ O-MecAMP sample.

O-Me-cAMP treatment (white arrow). Bar, $10 \mu \mathrm{M}$. Microtubules in HPAECs retrovirally transduced with GFP-tubulin elongate and appear more distinct (white arrow) after O-Me-cAMP treatment. 
complete media, low calcium conditions, or low calcium followed by calcium repletion. O-Me-cAMP treatment of HUVEC plated at low density, resulting in minimal cell-cell contact, also induced a significant increase in mean process length (Figure 3A). Thus, intact junctions are not required for Epac-induced changes in MT distribution. Next, the requirement for Rap activation in this process was evaluated. Rap1GAP-GFP overexpression in HUVECs resulted in a complete reduction in Rap1 and Rap2 GTP loading compared with GFP-expressing cells (Figure 3B). The O-MecAMP-induced increase in process length was similar in cells transduced with GFP or Rap1GAP, indicating that Epac regulation of Rap is not responsible for the increase in MT length (Figure 3C). Although Epac GEF-independent activation of JNK has been reported (Hochbaum et al., 2003), we did not observe JNK phosphorylation after treatment with O-Me-cAMP (data not shown). This suggests that the JNK pathway is not responsible for the Rap-independent modulation of MT dynamics by Epac.

An increase in MT length may result from increased growth, a decrease in catastrophic events and/or an increase in the capture of the growing ends (Gundersen et al., 2004). Here, we examined whether Epac activation influences MT growth. The MT binding protein EB3 associates specifically with the plus ends of growing MTs, and when expressed in cells as a GFP-fusion protein it can serve as a marker for visualizing MT growth in live cells (Stepanova et al., 2003). EB3-GFP was virally introduced into HUVECs, and movements of the GFP-tagged protein along the MT were visualized by fluorescence live-cell imaging. EB3-GFP occurred as comets that radiated from the MTOC and traveled toward the periphery of the cell. Treatment with O-Me-cAMP resulted in a significant increase in the average velocity of comet movement (Figure 4), indicating that Epac activation results in an increase in MT growth rate.

\section{An Intact MT Network Is Required for Epac-induced Increases in Cortical Actin but Not Epac-mediated Activation of Rap GTPase}

The majority of studies related to cross-talk between the actin cytoskeleton and MTs has been analyzed in the context of cell adhesion and migration (Small and Kaverina, 2003) rather than in the context of cell-cell contacts. To determine whether the O-Me-cAMP-induced actin cytoskeletal changes are dependent on an intact MT network, HUVECs were treated with vehicle or the MT-disrupting agent nocodazole before $\mathrm{O}-\mathrm{Me}-\mathrm{cAMP}$ stimulation. Actin arrangement was assessed by immunofluorescence and cell fractionation approaches. MT distribution was evaluated by $\beta$-tubulin staining. In control monolayers, MT and actin filaments spanned the cytosol. Treatment with a low dose of nocodazole resulted in a collapse of the MT network. Under these conditions the peripheral localized actin was greatly reduced and replaced by stress fibers. O-Me-cAMP treatment resulted in an increase in cortical actin (Figure 5A) in control monolayers but not nocodazole-treated monolayers. As expected, O-Me-cAMP had no effect on MT distribution in the presence of nocodazole. We postulated that increases in the observed organized actin-based structures, which was primarily cortical actin after O-MecAMP treatment, would be associated with an increase in actin-associated proteins in the insoluble cytoskeletal fraction. Therefore, cells were subjected to fractionation, and they were immunoblotted for the actin-associated motor nonmuscle myosin. O-Me-cAMP treatment increased nonmuscle myosin heavy chain in the cytoskeletal compared with the detergent-soluble cytosolic fraction in
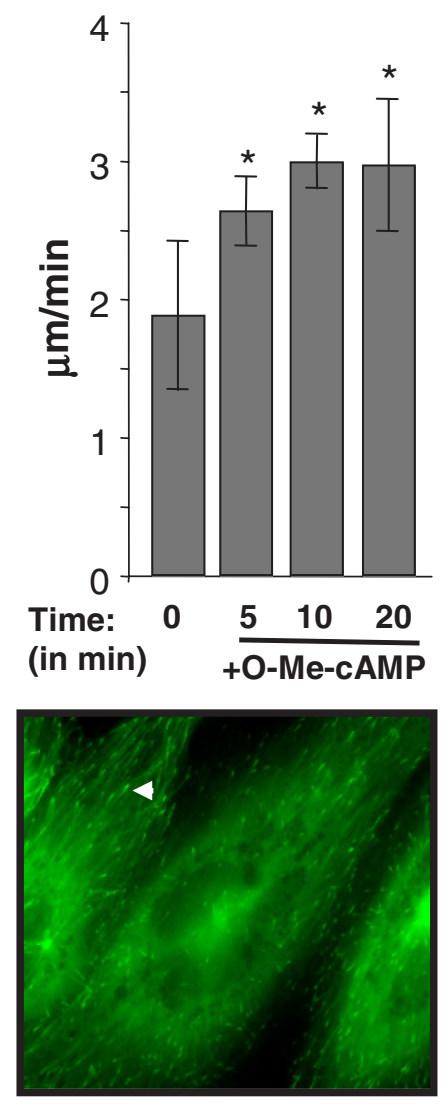

\begin{tabular}{|l|l|}
\hline $\begin{array}{l}\text { Time } \\
(\mathrm{min})\end{array}$ & $\begin{array}{c}\text { Fold } \\
\text { induction }\end{array}$ \\
\hline $\mathbf{0}$ & $1.0 \pm 0.00$ \\
\hline $\mathbf{5}$ & $1.40 \pm 0.10^{*}$ \\
\hline $\mathbf{1 0}$ & $1.43 \pm 0.18^{\star}$ \\
\hline $\mathbf{2 0}$ & $1.62 \pm 0.04^{*}$ \\
\hline
\end{tabular}

Figure 4. O-Me-cAMP treatment results in an increase in MT growth rate. Live-cell imaging of HUVECs was undertaken $48 \mathrm{~h}$ after infection with retroviruses expressing EB3-GFP, a plus-end MT marker. Twenty-four hours after infection, cells were placed in serum-free media, and samples were imaged before $(0 \mathrm{~min})$ and after addition of O-Me-cAMP for the indicated time points in minutes. The EB3-GFP comet distance traveled over time (in seconds) was calculated. One representative of three experiments is shown, and the average -fold induction in three to five independent experiments is given (Table). ${ }^{*} \mathrm{p}<0.05$ compared with 0 -min sample. A frame of a time-lapse sequence is shown to depict the EB3 localization on MT structures. EB3-GFP labels growing MT plus ends in a characteristic "comet" staining pattern that has a bright front and dim tail (arrowheads).

control cells (Figure 5B), indicating actin polymerization and redistribution of actin-associated proteins. These changes were not observed in cells pretreated with cytochalasin $\mathrm{D}$ treatment, thus validating the dependence of this redistribution on actin polymerization. The $\mathrm{O}-\mathrm{Me}-$ cAMP-induced localization of nonmuscle myosin to the cytoskeletal fraction was abrogated in nocodazole-treated 
A
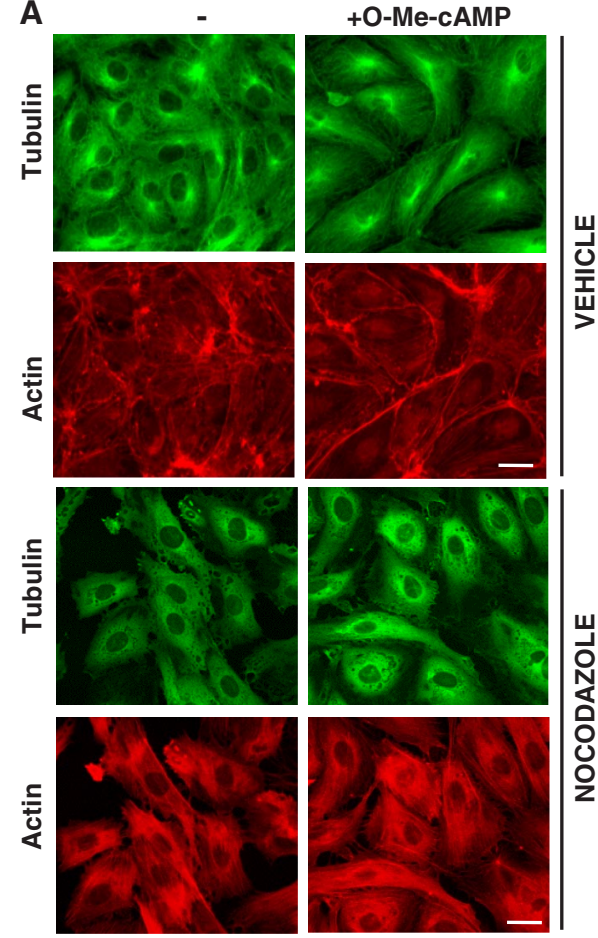

B

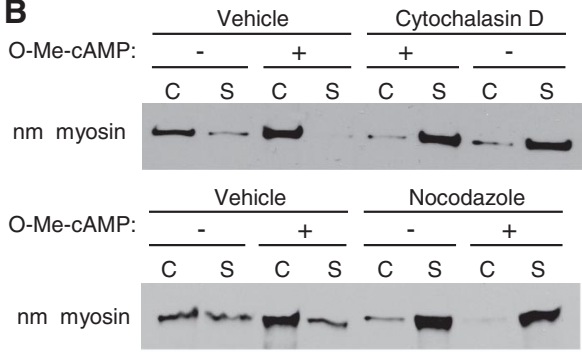

C

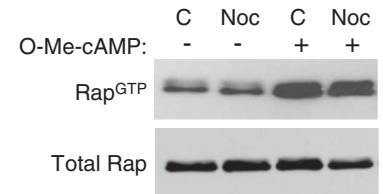

Figure 5. Analysis of O-Me-cAMP-induced F-actin and Rap1 activation after nocodazole treatment of HUVECs. (A) HUVEC were treated with vehicle alone or with $0.15 \mu \mathrm{M}$ nocodazole for $5 \mathrm{~min}$ and simultaneously treated with control (-) or O-Me-cAMP. Cells were fixed and stained with fluorescein isothiocyanate-labeled anti-tubulin antibody (microtubules) and rhodamine-phalloidin (F-actin). Nocodazole treatment prevented $\mathrm{O}-\mathrm{Me}-\mathrm{cAMP}$ induced $\mathrm{F}$-actin changes detected in control/O-Me-cAMP-treated cells. One of five representative experiments is shown. Bar, $10 \mu \mathrm{m}$. (B) The Triton-X cytosolic soluble (S) and cytoskeletal (C) fractions were prepared from endothelial cells treated with O-Me-cAMP, cytochalasin D, and nocodazole as indicated, and then they were immunoblotted with nonmuscle myosin heavy chain (nm myosin) antibody. (C) Pull-down assay of active Rap were performed on HUVECs incubated without $(-)$ or with $0.15 \mu \mathrm{M}$ nocodazole (+) for $5 \mathrm{~min}$ followed by a $20 \mathrm{~min}$ treatment with O-Me-cAMP $(+)$ as indicated. Western blots were probed for Rap1. One of three independent experiments is shown for $\mathrm{B}$ and one of two for $\mathrm{C}$.

cells. These data indicate that the observed Epac-induced increases in cortical actin require an intact MT network. Similar results were obtained following elevation of cAMP with forskolin and rollipram (data not shown).
To determine whether Epac-induced activation of Rap requires an intact MT network, Epac-mediated GTP loading of Rap1 was evaluated in the presence of nocodazole. HUVECs pretreated with nocodazole had levels of GTPbound Rap that were similar to those observed in untreated cells. O-Me-cAMP treatment resulted in an increase in Rap activation that was similar in untreated and nocodazole treated cells, suggesting that Rap activation does not per se rely on an intact MT network (Figure 5C). Together, these data suggest that Epac-induced Rap activation needs MT functions to exert its effects on the actin cytoskeleton.

\section{Epac-induced Enhancement of Barrier Function Requires a Dynamic Microtubule Network}

$\mathrm{O}-\mathrm{Me}-\mathrm{cAMP}$ treatment resulted in a rapid increase in electrical resistance, which reflects an increase in barrier function. Disruption of the actin cytoskeleton or the MT network with cytochalasin D and nocodazole, respectively, led to a rapid decrease in TER (Figure 6, A and B) at concentrations that resulted in gap formation but that did not lead to significant cell detachment as assessed by VE-cadherin staining of samples subjected to TER analysis (Figure 6C). O-MecAMP treatment failed to reverse these changes in TER (Figure 6, A and B). F + R also failed to reverse nocodazolemediated barrier dysfunction (Figure 6B). Thus, both cytoskeletal networks, required to maintain tight barrier function under basal conditions, are required for Epac-induced increases in barrier function. Depolymerization of MTs is known to activate RhoA that subsequently results in MLC phosphorylation (Ren et al., 1999; Birukova et al., 2004b). Activation of MLC has been associated with enhanced permeability leading to the possibility that the failure to reverse leakage after O-Me-cAMP or F+R treatment is the inability of these compounds to reverse the effects of MLC activation. Nocodazole increased MLC phosphorylation, which was not reversed by O-Me-cAMP (Figure 6B). However, this was reversed after $\mathrm{F}+\mathrm{R}$ treatment, likely through $\mathrm{PKA}$ activation (Birukova et al., 2004b). Despite this, the nocodazole-induced leakage remained unaffected (Figure 6B). Because neither $\mathrm{F}+\mathrm{R}$ nor $\mathrm{O}-\mathrm{Me}-\mathrm{cAMP}$ could rescue the increase in permeability after nocodazole treatment independently of their effect on MLC phosphorylation, their inability to reverse barrier dysfunction is likely directly related to their reliance on an intact MT network.

To solidify our model that Epac affects vascular permeability through MT dynamics, we have used the MT-stabilizing agent Taxol. Unlike nocodazole, stabilization of the MT network with Taxol had no effect on the basal permeability of the endothelial monolayer. Treatment of the monolayer with Taxol increased O-Me-cAMP-mediated barrier enhancement, which correlated with a reduction in actin stress fibers and a more pronounced delineation of actin at the junctions. However, at later time points Taxol interfered with the barrier-enhancing effects of O-Me-cAMP (Figure 6D). Taxol pretreatment partially reversed TNF-induced barrier disruption over time as reported previously (10). This suggests that MTs are playing dual roles in O-MecAMP-induced endothelial barrier function. Although MT stability may initially enhance O-Me-cAMP-induced barrier properties and actin reorganization and it may prevent TGF $\beta$ - and TNF- $\alpha$-induced vascular permeability, a dynamic MT network is essential for sustaining the enhanced barrier effects of O-Me-cAMP.

TNF- $\alpha$, thrombin, and TGF $\beta$-induced endothelial permeability is associated with the destabilization of the peripheral MT network (Petrache et al., 2003; Birukova et al., 2004a, 2005) that could be attenuated by stabilization of MTs. We 
Figure 6. Role of Epac activation in MT based changes in barrier function. TER was examined in HUVECs treated with vehicle (Veh) or $2.0 \mu \mathrm{M}$ cytochalasin D (Cyto D) (A) or $0.15 \mu \mathrm{M}$ nocodazole (Noc) (arrow; B) and subsequently minus (Con) or plus O-Me-cAMP or $\mathrm{F}+\mathrm{R}$ (dotted arrow). Pull-down assays for active Rap1 (Rap1 ${ }^{\mathrm{GTP}}$ ) were performed on HUVECs treated without $(-)$ or with $\mathrm{F}+\mathrm{R}$ or $\mathrm{O}-\mathrm{Me}-\mathrm{cAMP}$ for $20 \mathrm{~min}$ to demonstrate efficient stimulation of Rap in cells by these agents (right, top). Right, cell lysates of samples pretreated with vehicle $(-)$ or nocodazole (Noc) for $10 \mathrm{~min}$, and subsequently treated with O-Me-cAMP (O-Me) or F + R for $20 \mathrm{~min}$, were subjected to Western blot analysis by using an antibody to phosphoMLC2 (Ser19) and $\beta$-tubulin (loading control). (C) Representative pictures of samples subjected to TER analysis in A and B and subsequently stained with anti-VE-cadherin are shown. Bar, $10 \mu \mathrm{m}$. (D) TER was examined in endothelial monolayers treated with vehicle (Veh) or $10 \mu \mathrm{m}$ Taxol for $1 \mathrm{~h}$ as indicated by the solid arrow and subsequently with O-Me-cAMP (left) or $20 \mathrm{ng} / \mathrm{ml}$ TNF (right) as indicated by the dotted arrow. In parallel, Taxol- or vehicle-treated cells were stimulated with or without O-MecAMP for $20 \mathrm{~min}$ and immunostained with rhodamine-phalloidin to visualize actin. (E) TER was measured in HUVECs treated with vehicle alone or $20 \mathrm{ng} / \mathrm{ml}$ TNF (solid arrow) for $16 \mathrm{~h}$, after which O-Me-cAMP or vehicle control were added (dotted arrow). (F) TER was measured in HUVECs that were treated with vehicle alone or $2 \mathrm{ng} / \mathrm{ml}$ TGF $\beta$ (solid arrow) followed by the addition of control (Con) or O-Me-cAMP (dotted arrow). One representative of three experiments is shown for A-F.
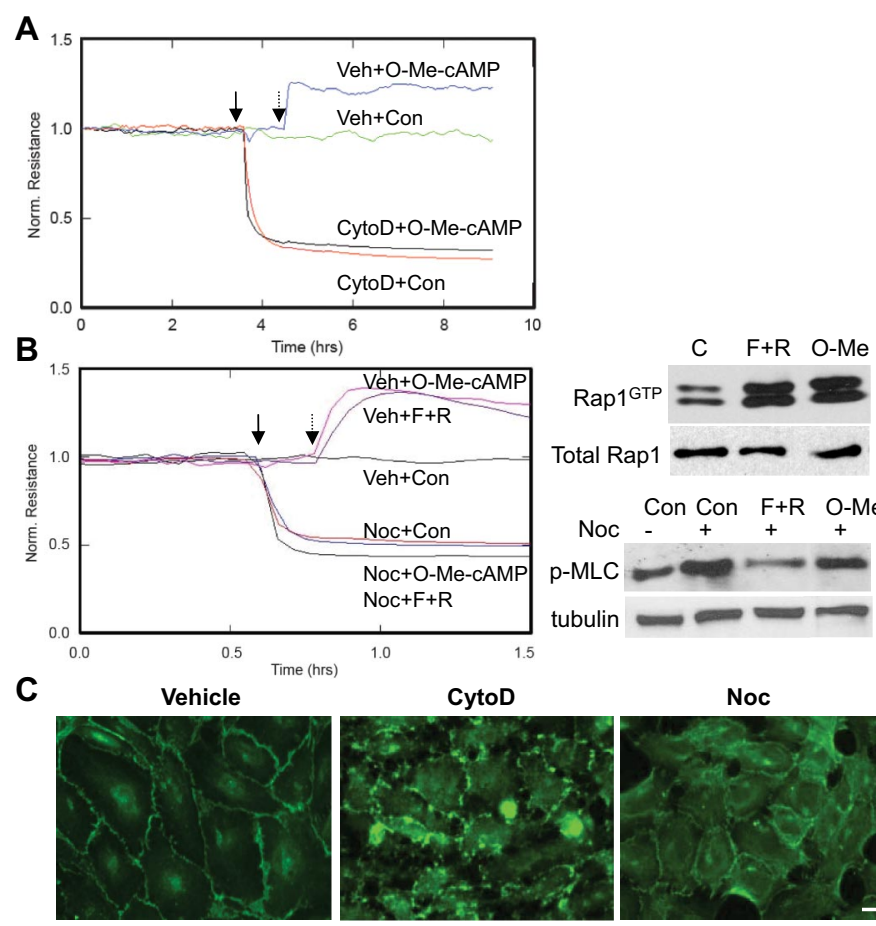

D
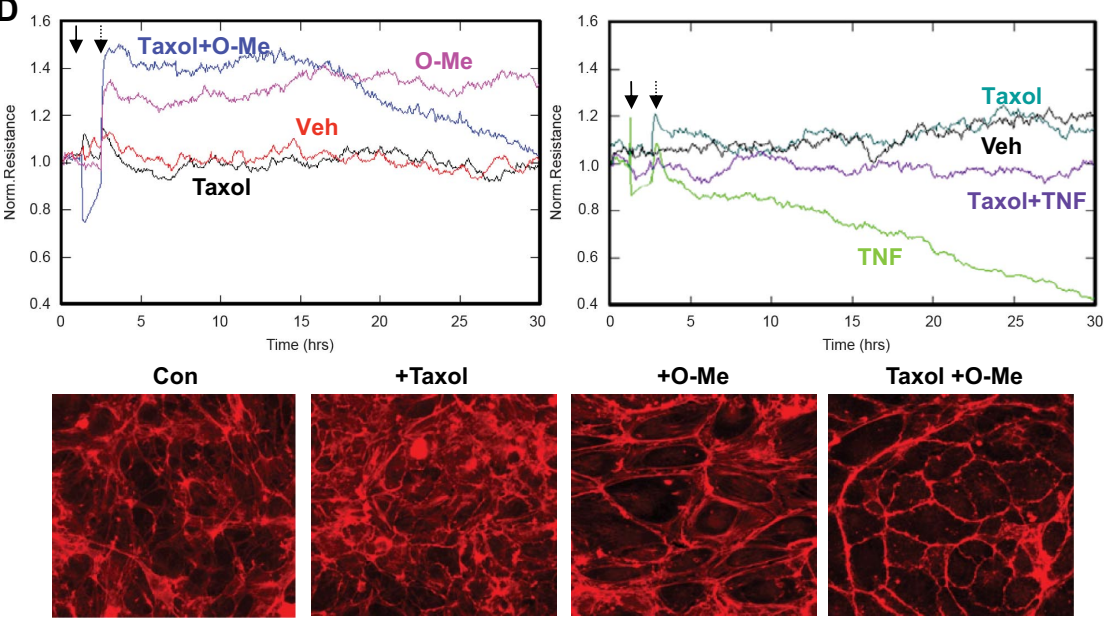

Taxol +O-Me

$\mathbf{E}_{1.5}$
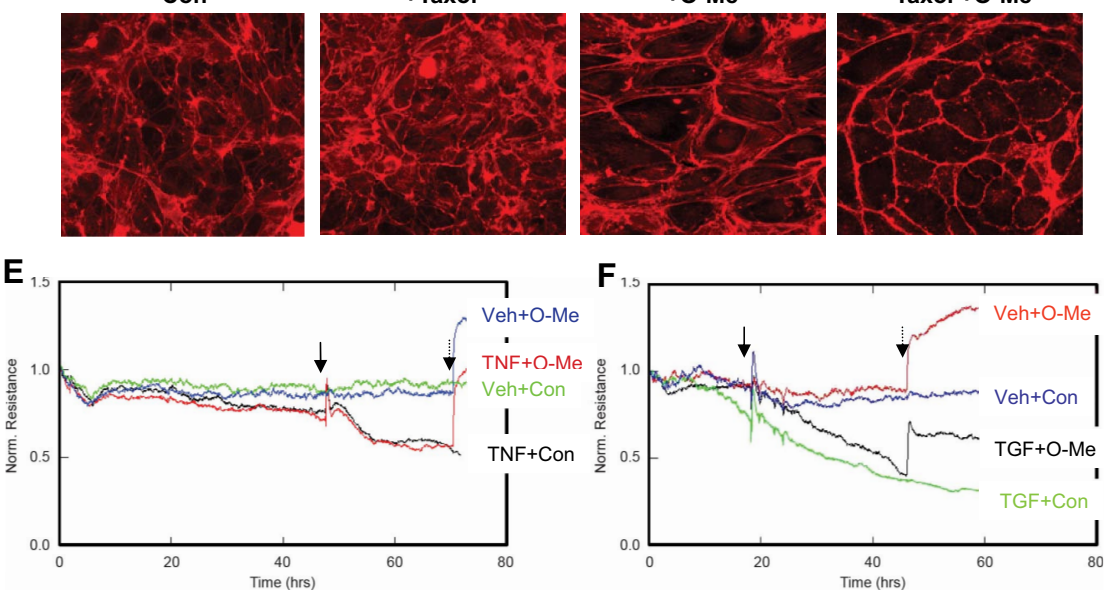

evaluated whether the observed Epac-mediated increase in MT growth correlated with an ability to reverse MT-dependent vascular permeability induced by TNF- $\alpha$ and TGF $\beta$. Treatment of HUVECs with TNF- $\alpha$ or TGF $\beta$ resulted in a time-dependent decrease in TER, indicating endothelial barrier compromise (Figure 6, E and F) as reported previously (Petrache et al., 2003; Birukova et al., 2005). The addition of $\mathrm{O}-\mathrm{Me}$-cAMP to these cytokine-treated cells significantly increased the TER (Figure 6C). These data suggest that Epac- mediated regulation of MT dynamics may underlie the observed reversal of TNF- $\alpha$ - and TGF $\beta$-induced vascular permeability.

\section{DISCUSSION}

Epacs are cAMP-responsive proteins implicated in a wide range of cellular responses, including mitosis, protein secretion, and integrin-mediated adhesion (Bos, 2003). In HUVECs, 
Epac activation of Rap GTPases enhances barrier function. Epac1 had a major role for O-Me-cAMP-induced changes in the cytoskeleton and MT process lengths. A role for Epac1 in changes in cortical actin was published previously, although Epac2 was not expressed in these endothelial cell cultures (Kooistra et al., 2005). Here, we provide evidence that Epac plays a significant role in regulating MTs and that this has implications for barrier integrity. Epac1 localized to MT structures, and its activation increased MT growth and net process lengths. This Epac function did not require Rap GTPases, and it was not dependent on cell-cell contacts. These data together with previous work indicate that Epac1 regulation of Rap GTPases enhances cortical actin, whereas Epac on MTs regulates MT dynamics independently of Rap GTPases. Epac regulation of MT may deliver Rap effectors that enhance cortical actin and subsequent VE-cadherin at cell-cell contacts (Figure 7). The effect of Epac activation on MT dynamics may have physiological consequences for barrier function as activating Epac reverses vascular permeability increased by TGF $\beta$ and TNF- $\alpha$, which are cytokines that promote barrier dysfunction by destabilizing MTs (Petrache et al., 2003; Birukova et al., 2004a, 2005).

Epac activation results in morphologically more discrete and linear MT filaments in HUVEC monolayers. The functional consequences of the increase in MT linearity is not clear, but data from migrating cells suggest that the orientation of MTs relative to the leading edge may be a reflection of the state of MT assembly/disassembly (Waterman-Storer et al., 2000). MTs perpendicular to the leading edge exhibited slower growth rates and more frequent catastrophes compared with MTs that were parallel to the leading edge. A similar analogy may apply to endothelial cells. Importantly, Epac positively influenced the rate of MT elongation and increased the overall MT process lengths in cells. This Epac function does not require Rap GTPases, suggesting direct effects of Epac on MT dynamics. We cannot rule out the possibility that increases in cell spreading, albeit small as the cells are in a confluent monolayer, may contribute to MT length. However, we reason that it is unlikely that integrinmediated cell spreading is primarily responsible for the increase in MT length because Epac-mediated integrin activation is well described to be Rap dependent (Bos, 2006), and here we show that MT dynamics do not rely on Rap activation. Recent studies demonstrated that Epac directly binds MAP1A-LC2, an interaction that promoted MT poly-

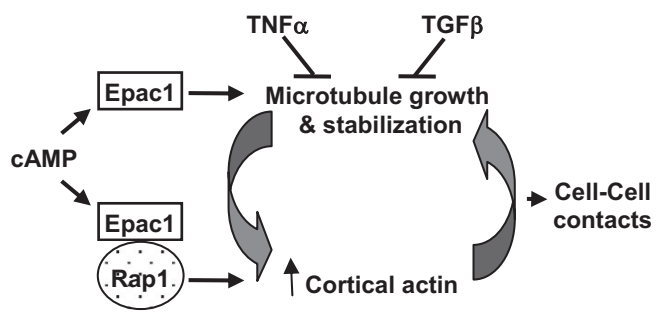

Figure 7. Model of Epac1-mediated effects on the MT and actin cytoskeleton required for endothelial junctional integrity. Elevations in cAMP results in Epac1 induced Rap activation with effects on cortical actin. Epac1 also promotes MT growth, in a Rap-independent manner, which is required for the Rap-dependent increases in cortical actin. Cortical actin in turn aids in MT stabilization by allowing greater tethering and capture of MT growing ends. The changes in cortical actin and MT dynamics are independent of VE-cadherin containing cell-cell interactions. TNF- $\alpha$ and TGF $\beta$ increase vascular permeability by destabilizing MTs. Epac activation counteracts the effects of these cytokines by promoting MT growth, stabilization, or both. merization in vitro (Mei and Cheng, 2005; Yarwood, 2005). A clear area for future investigation is to examine whether this interaction with MAP1A is responsible for the observed MT growth in HUVECs in response to Epac activation. The association of increased MT growth with a net increase in MT filament length in endothelial cells treated with O-MecAMP suggests that the Epac-induced growth of MT is coupled to increased capture of the growing ends at the periphery of cells. Studies in epithelial cells suggest that cell-cell interactions induce stabilization of MTs (Bre et al., 1990; Pepperkok et al., 1990; Waterman-Storer et al., 2000). Interestingly, cell-cell contact was not a prerequisite for O-Me-cAMP-induced increases in overall MT process lengths. Other compartments such as focal adhesions could be involved in tethering and capture of MTs (Gundersen et al., 2004). It is notable that the O-Me-cAMP-induced increase in cortical actin also does not require junctional VEcadherin (Cullere et al., 2005). Although VE-cadherin is not required for $\mathrm{O}-\mathrm{Me}-\mathrm{cAMP}$-induced changes in $\mathrm{MT}$ and actin polymerization, junctional VE-cadherin increases upon OMe-cAMP treatment (Cullere et al., 2005; Fukuhara et al., 2005), and it is required for tight barrier function (Sehrawat, unpublished data), as reported by others in in vivo and in vitro models (Dejana et al., 2000). Thus, MT and actin rearrangements induced by O-Me-cAMP may enhance VE-cadherin-mediated cell-cell contacts required for increased endothelial barrier function (Figure 7). The association of Epac with MTs even in the absence of O-Me-cAMP suggested the possibility that Epac also regulates MT under basal conditions. However, Epac siRNA-expressing cells did not exhibit a marked disruption of MTs compared with Luc siRNA control cells (Supplemental Figure 2S). Therefore, the effect of Epac siRNA in permeability in unstimulated conditions likely reflects its observed effects on cortical actin.

Epac regulation of Rap enhances cortical actin, whereas Epac-induced increases in MT process lengths is Rap independent. Descriptions of Epac functions independent of its activity as a Rap GEF are limited. Studies by Hochbaum et al., 2003 in 293 cells suggest that Epac activation of JNK is not dependent on Rap or the Epac catalytic GEF domain (Hochbaum et al., 2003). However, JNK is likely not downstream of Epac in our studies, because O-Me-cAMP failed to induce JNK phosphorylation in HUVECs (data not shown). An intact MT network was also not required for Epacinduced Rap activation, but it is sufficient to inhibit Epacinduced actin changes. Unlike our assays, a previous study showed that Epac-induced Rap activation was nocodazole sensitive (Gupta and Yarwood, 2005), which may reflect cell-type differences in sensitivity to nocodazole treatment with respect to Rap signaling pathways. In our assays, the morphological analysis of endothelial cells was done with concentrations of nocodazole that do not result in gross disruption of the monolayer, but they still significantly affect endothelial barrier properties. Under these conditions, Rap showed normal GTP loading after O-Me-cAMP treatment.

Our studies suggest a model wherein Epac on MTs regulates MT dynamics, whereas Epac activation of Rap GTPase pools present at junctions (Cullere et al., 2005) activates Rap effectors that are important in enhancing cortical actin (Figure 7). These effectors could include AF-6 (Boettner et al., 2000), an actin-binding protein that links junction-associated proteins such as zona occludens- 1 and nectin to the actin cytoskeleton, and/or GEFs for members of the Rho family of GTPases. Indeed, Rap has been shown to regulate the localization of Vav and Tiam1 (Arthur et al., 2004), GEFs for Rac and cdc42 which, in turn, have been implicated in endothelial junctional integrity (Wojciak-Stothard and Ridley, 2002; 
Broman et al., 2006). Epac may enhance the targeted delivery of these effectors to the cortex through MTs. Recently, an effector of Rap, RapL, was shown to associate with MTs in vascular endothelial cells, a localization that was not dependent on Rap1 (Fujita et al., 2005). Furthermore, in hematopoietic cells, RapL has been implicated in the trafficking of integrins to the plasma membrane. Thus, an intriguing hypothesis is that RapL mediates the delivery of junctional proteins through Epac-regulated MTs.

In endothelial cells, MT depolymerization is associated with barrier dysfunction (Verin et al., 2001; Birukova et al., 2004b) and pretreatment with cAMP-elevating or MT-stabilizing agents prevented this effect (Birukova et al., 2004b). However, prior disruption of MTs with nocodazole prevented the ability of cAMP elevation and Epac activation to restore the barrier integrity. This indicates that an intact MT network is a prerequisite for these pathways to enhance barrier function. Importantly, the observation that MT stabilization with Taxol initially enhances barrier properties of $\mathrm{O}-\mathrm{Me}$-cAMP, whereas longer exposure induced barrier disruption provides evidence that a dynamic MT network is needed in Epac-induced barrier regulation. Epac activation reversed both TNF- $\alpha$ - and TGF $\beta$-induced vascular permeability, suggesting that MT destabilization by these agonists (Petrache et al., 2003; Birukova et al., 2005) may be reversed upon Epac activation (Figure 7). Interestingly, TNF treatment increased PDE2 activity, resulting in reduced intracellular cAMP levels (Seybold et al., 2005). Given our data, we speculate that this may result in a reduction in Epac activation and subsequent Rap activity leading to increased permeability.

In summary, this study demonstrated a primary role for Epac in regulating MT growth and subsequent productive increases in MT length in endothelial cells. Our results show that the MT and actin networks are functionally interconnected to promote barrier integrity and that Epac is a critical regulator of both cytoskeletal structures. The Epac-Rap pathways leading to cortical actin rearrangement and the Rap-independent Epac regulation of MTs may coordinately act to promote barrier function (Figure 7). Elucidation of the mechanism of Epac regulation of MT may lead to potential therapies for edema in acute vascular syndromes.

\section{ACKNOWLEDGMENTS}

We thank Peter O'Donnell for excellent technical assistance with imaging. Dr. Niels Galjart for the EB3-GFP construct, Dr. Xiaodong Cheng for Epac1-YFP cDNA, Dr. Patrick J. Casey, for the RapGAP1 adenovirus, and Michael Lauterbach for help with statistical analysis. This work was supported by National Institutes of Health grant P01 HL-036028 (to T.N.M.) and R01 HL-68130 (to J.I.). X.C. was supported by KO1AR054984, and S.P. was supported by training grant HL-066978 (L. Silberstein) and a Ruth L. Kirschstein fellowship HL082133-01.

\section{REFERENCES}

Arthur, W. T., Quilliam, L. A., and Cooper, J. A. (2004). Rap1 promotes cell spreading by localizing Rac guanine nucleotide exchange factors. J. Cell Biol. $167,111-122$.

Birukova, A. A., Birukov, K. G., Adyshev, D., Usatyuk, P., Natarajan, V., Garcia, J. G., and Verin, A. D. (2005). Involvement of microtubules and Rho pathway in TGF-beta1-induced lung vascular barrier dysfunction. J. Cell Physiol. 204, 934-947.

Birukova, A. A., Birukov, K. G., Smurova, K., Adyshev, D., Kaibuchi, K., Alieva, I., Garcia, J. G., and Verin, A. D. (2004a). Novel role of microtubules in thrombin-induced endothelial barrier dysfunction. FASEB J. 18, 1879-1890.

Birukova, A. A., Liu, F., Garcia, J. G., and Verin, A. D. (2004b). Protein kinase A attenuates endothelial cell barrier dysfunction induced by microtubule disassembly. Am. J. Physiol. Lung Cell Mol. Physiol. 287, L86-L93.
Birukova, A. A. et al. (2004c). Microtubule disassembly induces cytoskeletal remodeling and lung vascular barrier dysfunction: role of Rho-dependent mechanisms. J. Cell. Physiol. 201, 55-70.

Boettner, B., Govek, E. E., Cross, J., and Van Aelst, L. (2000). The junctional multidomain protein AF-6 is a binding partner of the Rap1A GTPase and associates with the actin cytoskeletal regulator profilin. Proc. Natl. Acad. Sci. USA 97, 9064-9069.

Borbiev, T., Verin. A. D., Shi, S., Liu, F., and Garcia, J. G. (2001). Regulation of endothelial cell barrier function by calcium/calmodulin-dependent protein kinase II. Am. J. Physiol. Lung Cell Mol. Physiol. 280, L983-L990.

Bos, J. L. (2003). Epac: a new cAMP target and new avenues in cAMP research Nat. Rev. Mol. Cell Biol. 4, 733-738.

Bos, J. L. (2006). Epac proteins: multi-purpose cAMP targets. Trends Biochem. Sci. 31, 680-686.

Bre, M. H., Pepperkok, R., Hill, A. M., Levilliers, N., Ansorge, W., Stelzer, E. H., and Karsenti, E. (1990). Regulation of microtubule dynamics and nucleation during polarization in MDCK II cells. J. Cell Biol. 111, 3013-3021.

Broman, M. T., Kouklis, P., Gao, X., Ramchandran, R., Neamu, R. F., Minshall, R. D., and Malik, A. B. (2006). Cdc42 regulates adherens junction stability and endothelial permeability by inducing alpha-catenin interaction with the vascular endothelial cadherin complex. Circ. Res. 98, 73-80.

Chi, J. T. et al. (2003). Endothelial cell diversity revealed by global expression profiling. Proc. Natl. Acad. Sci. USA 100, 10623-10628.

Cullere, X., Shaw, S. K., Andersson, L., Hirahashi, J., Luscinskas, F. W., and Mayadas, T. N. (2005). Regulation of vascular endothelial barrier function by Epac, a cAMP-activated exchange factor for Rap GTPase. Blood 105, 19501955.

de Rooij, J., Zwartkruis, F. J., Verheijen, M. H., Cool, R. H., Nijman, S. M., Wittinghofer, A., and Bos, J. L. (1998). Epac is a Rap1 guanine-nucleotideexchange factor directly activated by cyclic AMP. Nature 396, 474-477.

Dejana, E., Lampugnani, M. G., Martinez-Estrada, O., and Bazzoni, G. (2000). The molecular organization of endothelial junctions and their functional role in vascular morphogenesis and permeability. Int. J. Dev. Biol. 44, 743-748

Fang, Y., and Olah, M. E. (2007). Cyclic AMP-dependent, protein kinase A-independent activation of extracellular signal-regulated kinase $1 / 2$ following adenosine receptor stimulation in human umbilical vein endothelial cells: role of exchange protein activated by cAMP 1 (Epac1). J. Pharmacol. Exp. Ther. 322, 1189-1200.

Fujita, H., Fukuhara, S., Sakurai, A., Yamagishi, A., Kamioka, Y., Nakaoka, Y., Masuda, M., and Mochizuki, N. (2005). Local activation of Rap1 contributes to directional vascular endothelial cell migration accompanied by extension of microtubules on which RAPL, a Rap1-associating molecule, localizes. J. Biol. Chem. 280, 5022-5031.

Fukuhara, S., Sakurai, A., Sano, H., Yamagishi, A., Somekawa, S., Takakura, N., Saito, Y., Kangawa, K., and Mochizuki, N. (2005). Cyclic AMP potentiates vascular endothelial cadherin-mediated cell-cell contact to enhance endothelial barrier function through an Epac-Rap1 signaling pathway. Mol. Cell. Biol. $25,136-146$

Gundersen, G. G., Gomes, E. R., and Wen, Y. (2004). Cortical control of microtubule stability and polarization. Curr. Opin. Cell Biol. 16, 106-112.

Gupta, M., and Yarwood, S. J. (2005). MAP1A light chain 2 interacts with exchange protein activated by cyclic AMP 1 (EPAC1) to enhance Rap1 GTPase activity and cell adhesion. J. Biol. Chem. 280, 8109-8116.

Hochbaum, D., Tanos, T., Ribeiro-Neto, F., Altschuler, D., and Coso, O. A (2003). Activation of JNK by Epac is independent of its activity as a Rap guanine nucleotide exchanger. J. Biol. Chem. 278, 33738-33746.

Hong, J., Doebele, R. C., Lingen, M. W., Quilliam, L. A., Tang, W. J., and Rosner, M. R. (2007). Anthrax edema toxin inhibits endothelial cell chemotaxis via Epac and Rap1. J. Biol. Chem. 282, 19781-19787.

Kawasaki, H., Springett, G. M., Mochizuki, N., Toki, S., Nakaya, M., Matsuda, M., Housman, D. E., and Graybiel, A. M. (1998). A family of cAMP-binding proteins that directly activate Rap1. Science 282, 2275-2279.

Kooistra, M. R., Corada, M., Dejana, E., and Bos, J. L. (2005). Epac1 regulates integrity of endothelial cell junctions through VE-cadherin. FEBS Lett. 579, 4966-4972.

Ligon, L. A., Karki, S., Tokito, M., and Holzbaur, E. L. (2001). Dynein binds to beta-catenin and may tether microtubules at adherens junctions. Nat. Cell Biol. 3, 913-917.

Mei, F. C., and Cheng, X. (2005). Interplay between exchange protein directly activated by cAMP (Epac) and microtubule cytoskeleton. Mol. Biosyst. 1, 325-331. 
Noiges, R., Eichinger, R., Kutschera, W., Fischer, I., Nemeth, Z., Wiche, G., and Propst, F. (2002). Microtubule-associated protein 1A (MAP1A) and MAP1B: light chains determine distinct functional properties. J. Neurosci. 22, 2106-2114.

Patel, S. R., Richardson, J. L., Schulze, H., Kahle, E., Galjart, N., Drabek, K., Shivdasani, R. A., Hartwig, J. H., and Italiano, J. E., Jr. (2005). Differential roles of microtubule assembly and sliding in proplatelet formation by megakaryocytes. Blood 106, 4076-4085.

Pepperkok, R., Bre, M. H., Davoust, J., and Kreis, T. E. (1990). Microtubules are stabilized in confluent epithelial cells but not in fibroblasts. J. Cell Biol. 111, 3003-3012.

Petrache, I., Birukova, A., Ramirez, S. I., Garcia, J. G., and Verin, A. D. (2003). The role of the microtubules in tumor necrosis factor-alpha-induced endothelial cell permeability. Am. J. Respir. Cell. Mol. Biol. 28, 574-581.

Ren, X. D., Kiosses, W. B., and Schwartz, M. A. (1999). Regulation of the small GTP-binding protein Rho by cell adhesion and the cytoskeleton. EMBO J. 18, $578-585$

Seite, R., Leonetti, J., Luciani-Vullet, J., and Vio, M. (1977). Cyclic AMP and ultrastructural organization of the nerve cell nucleus: stimulation of nuclear microtubules and microfilaments assembly in sympathetic neurons. Brain Res. 124, 41-51.

Seybold, J. et al. (2005). Tumor necrosis factor-alpha-dependent expression of phosphodiesterase 2, role in endothelial hyperpermeability. Blood 105, 35693576 .

Small J. V, and Kaverina I. (2003). Microtubules meet substrate adhesions to arrange cell polarity. Curr. Opin. Cell Biol. 15, 40-47.

Stepanova, T., Slemmer, J., Hoogenraad, C. C., Lansbergen, G., Dortland, B., De Zeeuw, C. I., Grosveld, F., van Cappellen, G., Akhmanova, A., and Galjart, N. (2003). Visualization of microtubule growth in cultured neurons via the use of EB3-GFP (end-binding protein 3-green fluorescent protein). J. Neurosci. $23,2655-2664$

Tudor, E. L. et al. (2005). ALS2/Alsin regulates. Rac-PAK signaling and neurite outgrowth. J. Biol. Chem. 280, 34735-34740. van Hinsbergh, V.W.M., and van Nieuw Amerongen, G. P. (2002). Intracellular signalling involved in modulating human endothelial barrier function. J. Anat. 200, 549-560.

Verin, A. D., Birukova, A., Wang, P., Liu, F., Becker, P., Birukov, K., and Garcia, J. G. (2001). Microtubule disassembly increases endothelial cell barrier dysfunction: role of MLC phosphorylation. Am. J. Physiol. Lung Cell Mol, Physiol. 281, L565-L574.

Watanabe, T., Noritake, J., and Kaibuchi, K. (2005). Regulation of microtubules in cell migration. Trends Cell Biol. 15, 76-83.

Waterman-Storer, C. M., Salmon, W. C., and Salmon, E. D. (2000). Feedback interactions between cell-cell adherens junctions and cytoskeletal dynamics in newt lung epithelial cells. Mol. Biol. Cell 11, 2471-2483.

Wittchen, E. S., van Buul, J. D., Burridge, K., and Worthylake, R. A. (2005a). Trading spaces: Rap, Rac, and Rho as architects of transendothelial migration. Curr. Opin. Hematol. 12, 14-21.

Wittchen, E. S., Worthylake, R. A., Kelly, P., Casey, P. J., Quilliam, L. A., and Burridge, K. (2005b). Rap1 GTPase inhibits leukocyte transmigration by promoting endothelial barrier function. J. Biol. Chem. 280, 11675-11682.

Wojciak-Stothard, B., and Ridley, A. J. (2002). Rho GTPases and the regulation of endothelial permeability. Vascul. Pharmacol. 39, 187-199.

Woo, S, and Gomez TM. (2006). Rac1 and RhoA promote neurite outgrowth through formation and stabilization of growth cone point contacts. J. Neurosci. $26,1418-1428$

Yanagisawa, M., Kaverina, I. N., Wang, A., Fujita, Y., Reynolds, A. B., and Anastasiadis, P. Z. (2004). A novel interaction between kinesin and p120 modulates p120 localization and function. J. Biol. Chem. 279, 9512-9521.

Yarwood, S. J. (2005). Microtubule-associated proteins (MAPs) regulate cAMP signalling through exchange protein directly activated by cAMP (EPAC) Biochem. Soc. Trans. 33, 1327-1329.

Yuan, S. Y. (2003). Protein kinase signaling in the modulation of microvascular permeability. Vascul. Pharmacol. 39, 213-223. 